\title{
STATUS OF THYROID PROFILE IN TYPE-2 DIABETES MELLITUS
}

\author{
Shekhar Chandra Yadav, Alwin Saldhana and Biswajit Majumdar
}

\begin{abstract}
:
Diabetes mellitus is a group of metabolic disorder characterized by hyperglycemia resulting from defect in insulin secretion and/or insulin action. Thyroid dysfunction in diabetes mellitus patient has been frequently encountered. The clinical relationship between diabetes mellitus and thyroid function is becoming more widely recognized with hypothyroidism among diabetes mellitus patient. Total of 100 cases and 50 controls are enrolled into the study. Their fasting venous blood sample was taken and analyzed for blood sugar, T3, T4 and TSH. Blood sugar and TSH level were elevated while T3 and T4 level were statically decreased in case group in comparison to control group.
\end{abstract}

Key Words: Diabetes mellitus, hypothyroidism

\section{Introduction:}

Diabetes is regarded as single disease entity. Diabetes is a heterogeneous group of diseases characterized by chronic hyperglycemia resulting from diverse group of etiology such as environmental and genetic factors acting simultaneously or jointly.

The cause of Diabetes Mellitus is defective production of Insulin or defective action of Insulin, a hormone that controls the metabolism of carbohydrates, proteins and lipids. Diabetes is regarded as long term disease without variable clinical manifestation and progression of diseases. Chronic hyperglycemia from any route of cause leads to dyslipidemia, hypothyroidism and elevated thyroid stimulating hormone, cardiovascular diseases, renal diseases, neurological problems and recurrent infections. ${ }^{1}$

Diabetes mellitus is a group of metabolic disorders characterized by hyperglycemia resulting from defect in Insulin secretion and
Insulin action or both. Thyroid dysfunction in diabetes mellitus patients has been frequently encountered. The clinical relationship between Diabetes Mellitus and thyroid functions is becoming more widely recognized with hypothyroidism among diabetes mellitus patients. ${ }^{2}$

Diabetes is a "the bergh" disease affecting at least 20 million people throughout the world. Its prevalence in adult population is $2-5 \%$. In some developing countries such as Philippines, the disease prevalence is increasing rapidly due to rapid change in life style. IDDM effect 1 to 500 children and 1 in 200 adolescence. $^{3}$

Type 2 diabetes mellitus, epidemic in Indian is a result of social influence and changing life style. Epidemiological study in the 1960 s and 1970s using random and post load glucose estimation reported diabetes prevalence varying from $1-4 \%$ in the urban population and $1-2 \%$ the rural population and more standardize epidemiological study in 1990's reported prevalence rate that vary from $5-15 \%$ among urban population, $4-6 \%$ 


\section{Original Article}

in the semi-urban population and $1-5 \%$ in rural population. ${ }^{4}$

\section{Thyroid dysfunction:}

\section{Thyroid and thyroid dysfunction:}

Thyroid gland is unique among the organ of endocrine system because of its size and superficial location. It consists of two bulky lateral lobes connected by a relatively thin isthmus usually located below and anterior to the pharynx. The weight of normal adult thyroid is approximately $15-20 \mathrm{gm}$. The thyroid has rich intra glandular capillary network that is supplied by the superior inferior thyroid arteries.

The major hormone secreted is T3 and T4. Hyperthyroidism and hypothyroidism are two primary pathological conditions that involve the thyroid gland. ${ }^{5}$

There are few studies on type- 2 diabetes mellitus and thyroid dysfunction. They seem to indicate an assurance of thyroid dysfunction among diabetic when compared to the general population $(12.7 \%)$ where hypothyroidism is the most common type of dysfunction. They found no significant difference between patient thyroid profile and that of euthyroid patient with type- 2 diabetes mellitus. The highest percentage of patient whose thyroid dysfunction was diagnosed may justify routine thyroid function assessment of type-2 diabetes mellitus. ${ }^{6}$

\section{Material and Method:}

The present study was carried out on total 150 subjects. subjects were divided into two groups. First case group which consist of 100 subjects with known type-2 diabetes mellitus. And second control group which consist of 50 subjects who had no complain
Thyroid profile and Diabetes mellitus

and history of diabetes and any thyroid disease.

Twelve hours overnight fasting venous blood sample collected from these subject in fluoride and heparin vacutainer and samples were centrifuged for the estimation of Fasting Blood Sugar (FBS), T3, T4 and Thyroid Stimulating Hormone (TSH).

Sample was analyzed by using biochemistry autoanalyzer. BS-300 chemistry analyzer (Mindray) for analysis of fasting blood sugar and Advia centaur CB immunoassay system ( By Bayer) for analysis of hormones T3, T4 and TSH. FBS was estimated by GODPOD, Enzymatic photometric method while Chemiluminiscence method was used to estimate the levels of T3, T4 and TSH.

\section{Results:}

Present study analysed the fasting blood sugar and thyroid profile (T3,T4 and TSH) in Type-2 diabetes mellitus compared with control population. In this study two groups population was there; case group (51 male \& 49 female) and control group (26 male \& 24 female) having similar age and sex. The mean standard deviation of age and sex of case and control group is statically insignificant, shown in table 1 . The $\mathrm{P}$ value is $0.270 \mathrm{of}$ age and sex distribution of the study. The personal history consisting of diet, use of alcohol along with family history of the subjects was recorded.

In the thyroid profile T3 and T4 level is statically reduced in case group compare to control group which is shown in table 3 with $P$ value less than 0.001 respectively

But TSH value statically increased in case group compare to control group which is shown in table 3 and $P$ value is 0.001 . The level of fasting blood sugar is statically 


\section{Original Article}

elevated in case group compare to control group ( $\mathrm{P}$ value is less than 0.001 ) which is
Thyroid profile and Diabetes mellitus

shown in table 3.

Table 1: Age distribution of subjects studied

\begin{tabular}{|l|c|c|c|c|}
\hline \multirow{2}{*}{ Age in years } & \multicolumn{2}{|c|}{ Controls } & \multicolumn{2}{c|}{ Cases } \\
\cline { 2 - 5 } & No & $\%$ & No & $\%$ \\
\hline Up to 30 years & 1 & 2.0 & 1 & 1.0 \\
31-40 years & 12 & 24.0 & 19 & 19.0 \\
41-50 years & 22 & 44.0 & 35 & 35.0 \\
51-60 years & 7 & 14.0 & 28 & 28.0 \\
61-70 years & 6 & 12.0 & 13 & 13.0 \\
>70 years & 2 & 4.0 & 6 & 6.0 \\
Total & 50 & 100.0 & 100 & 100.0 \\
\hline Mean \pm SD & \multicolumn{2}{|c|}{$49.24 \pm 11.01$} & $51.34 \pm 10.92$ \\
\hline
\end{tabular}

Samples are age matched with $\mathrm{P}=0.270$

Figure1: Age distribution of subjects studied

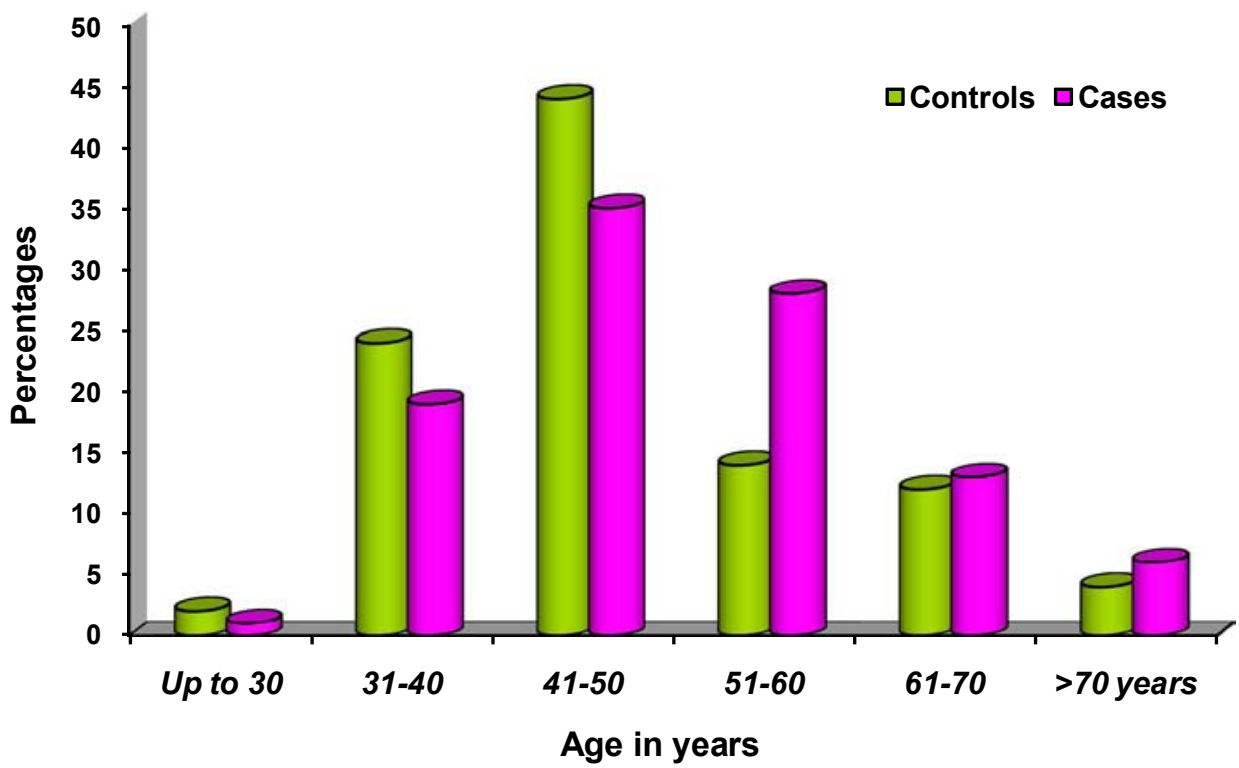


Table 2: Gender distribution of subjects studied

\begin{tabular}{|l|c|c|c|c|}
\hline $\begin{array}{l}\text { Gender Table 2: } \\
\text { Gender distribution } \\
\text { of subjects studied }\end{array}$ & \multicolumn{2}{|c|}{ Controls } & \multicolumn{2}{c|}{ Cases } \\
\cline { 2 - 5 } & No & $\%$ & No & $\%$ \\
\hline Male & 26 & 52.0 & 51 & 51.0 \\
Female & 24 & 48.0 & 49 & 49.0 \\
Total & 50 & 100.0 & 100 & 100.0 \\
\hline
\end{tabular}

Samples are gender matched with $\mathrm{P}=0.908$

Figure 2: Gender distribution of subjects studied
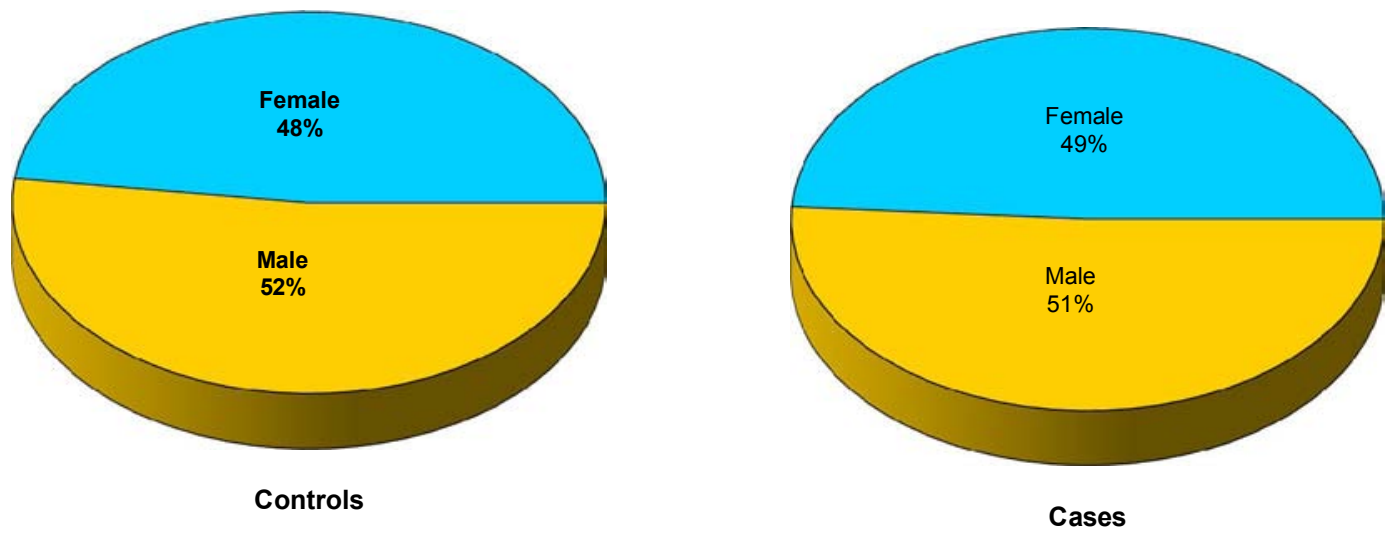
Table 3: Comparison of levels of sugar and thyroid parameters Between controls and cases

\begin{tabular}{|l|c|c|c|c|}
\hline Parameters & Controls & Cases & P value & Effect size \\
\hline \multirow{2}{*}{ FBS (mg/dl) } & $91.14 \pm 9.46$ & $160.75 \pm 44.93$ & $<0.001 * *$ & $1.86(\mathrm{VL})$ \\
& $(63-109)$ & $(112-350)$ & & \\
& $1.49 \pm 0.26$ & $0.60 \pm 0.44$ & $<0.001 * *$ & $2.27(\mathrm{VL})$ \\
& $(0.91-2.00)$ & $(0.01-2.00)$ & & \\
T3 & $7.82 \pm 2.49$ & $3.79 \pm 1.92$ & $<0.001 * *$ & $1.89(\mathrm{VL})$ \\
& $(3.40-12.30)$ & $(0.20-11.20)$ & & \\
TSH & $2.89 \pm 1.33$ & $10.38 \pm 7.73$ & $<0.001 * *$ & $1.17(\mathrm{~L})$ \\
& $(0.35-6.06)$ & $(3.90-62.45)$ & & \\
\hline
\end{tabular}

Figure 3: Comparison of levels of sugar parameters between controls and cases

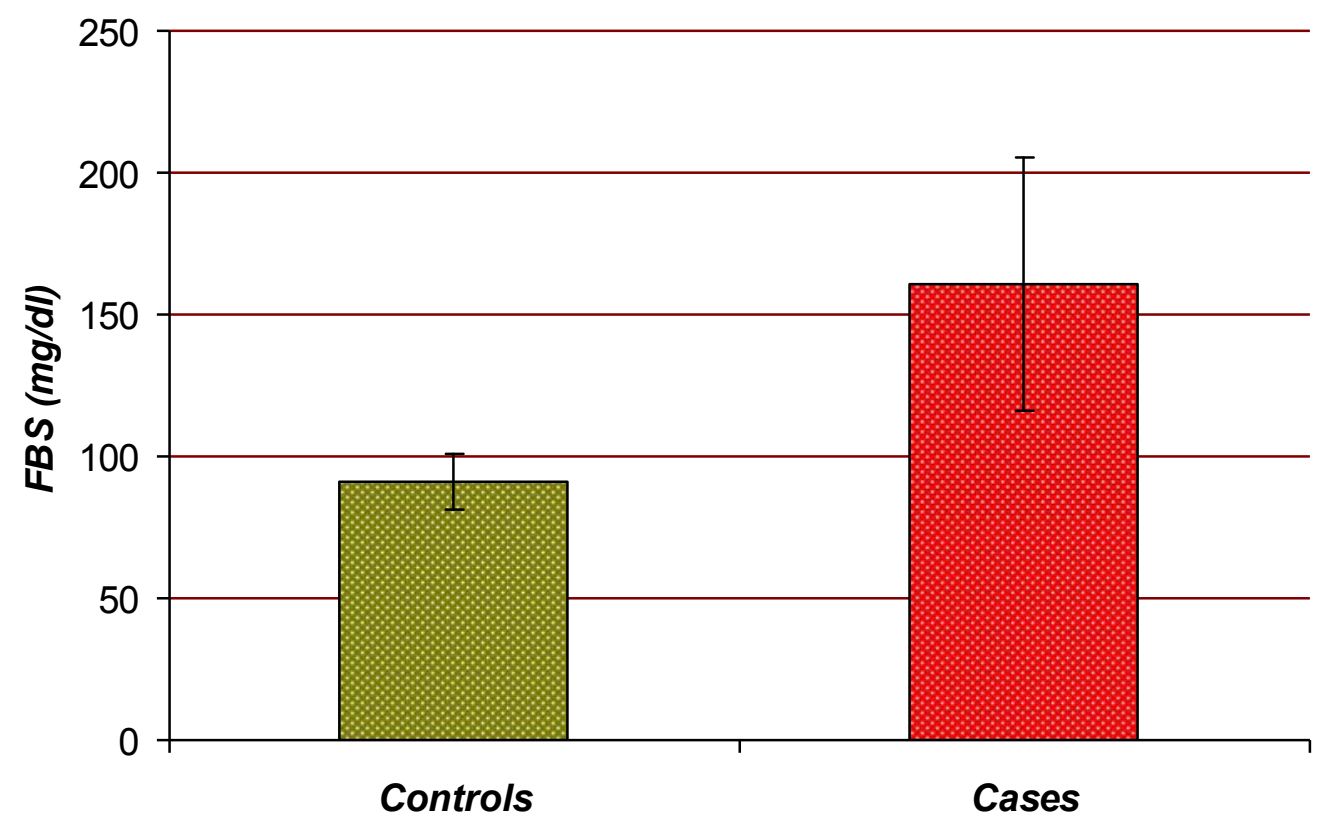


Figure 4A: Comparison of levels of $\mathrm{T} 3$ between controls and cases

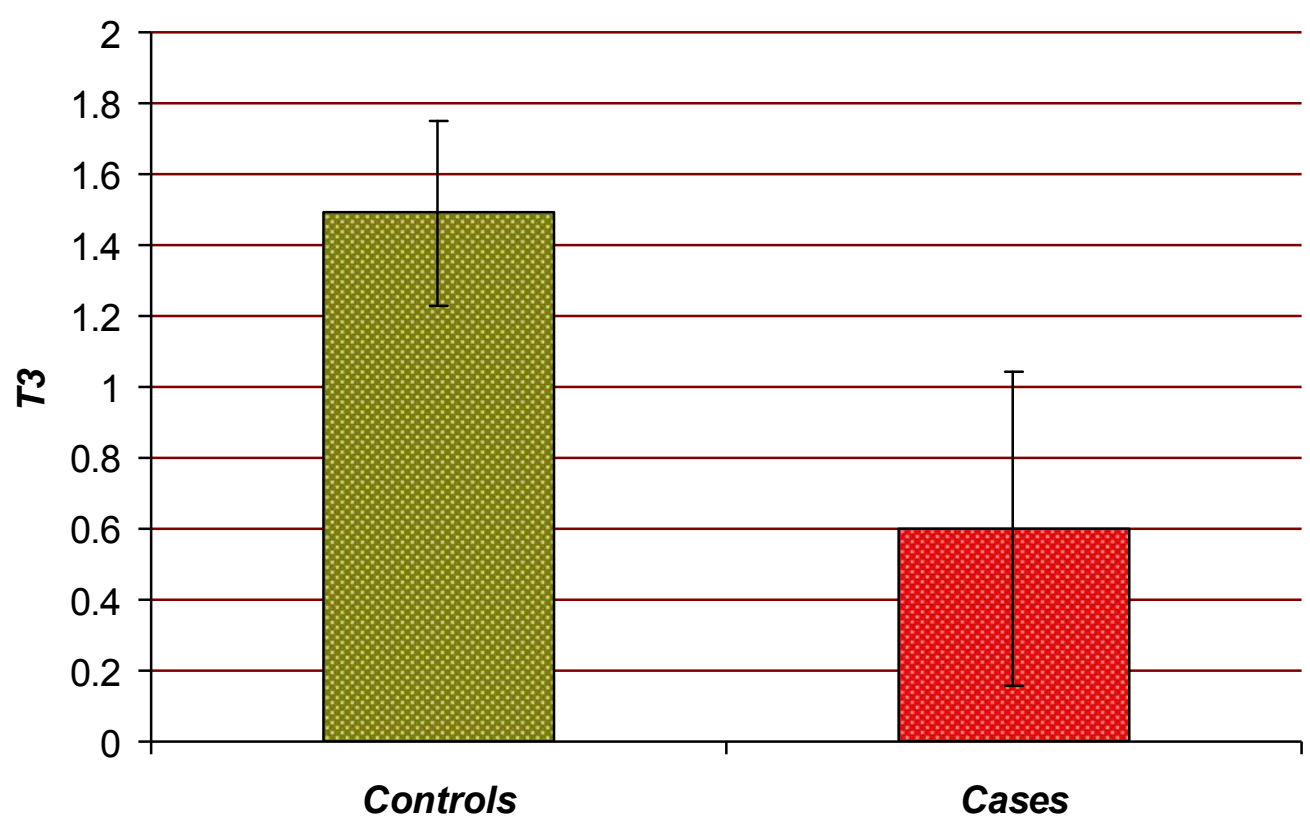

Figure 4B: Comparison of levels of $\mathrm{T} 4$ between controls and cases

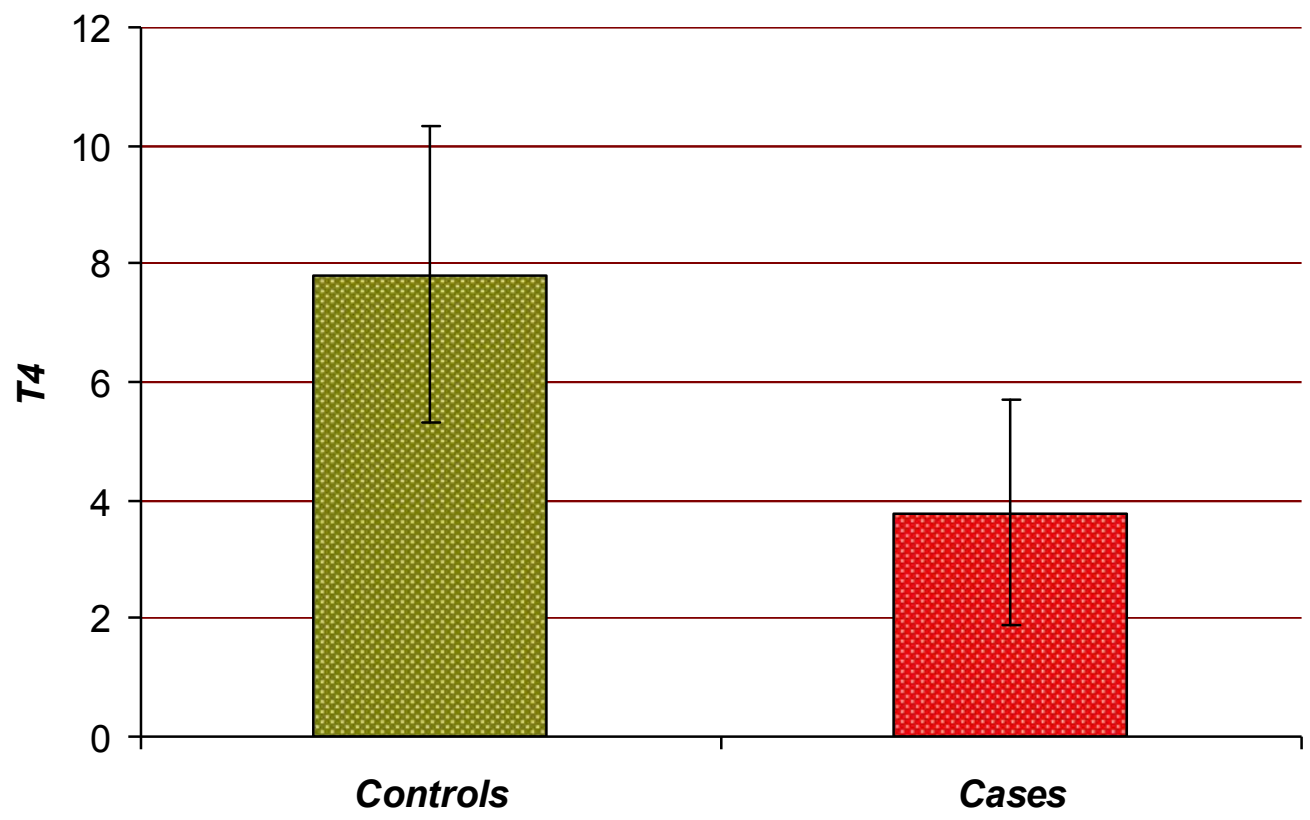


Figure 4C: Comparison of levels of TSH between Controls and cases

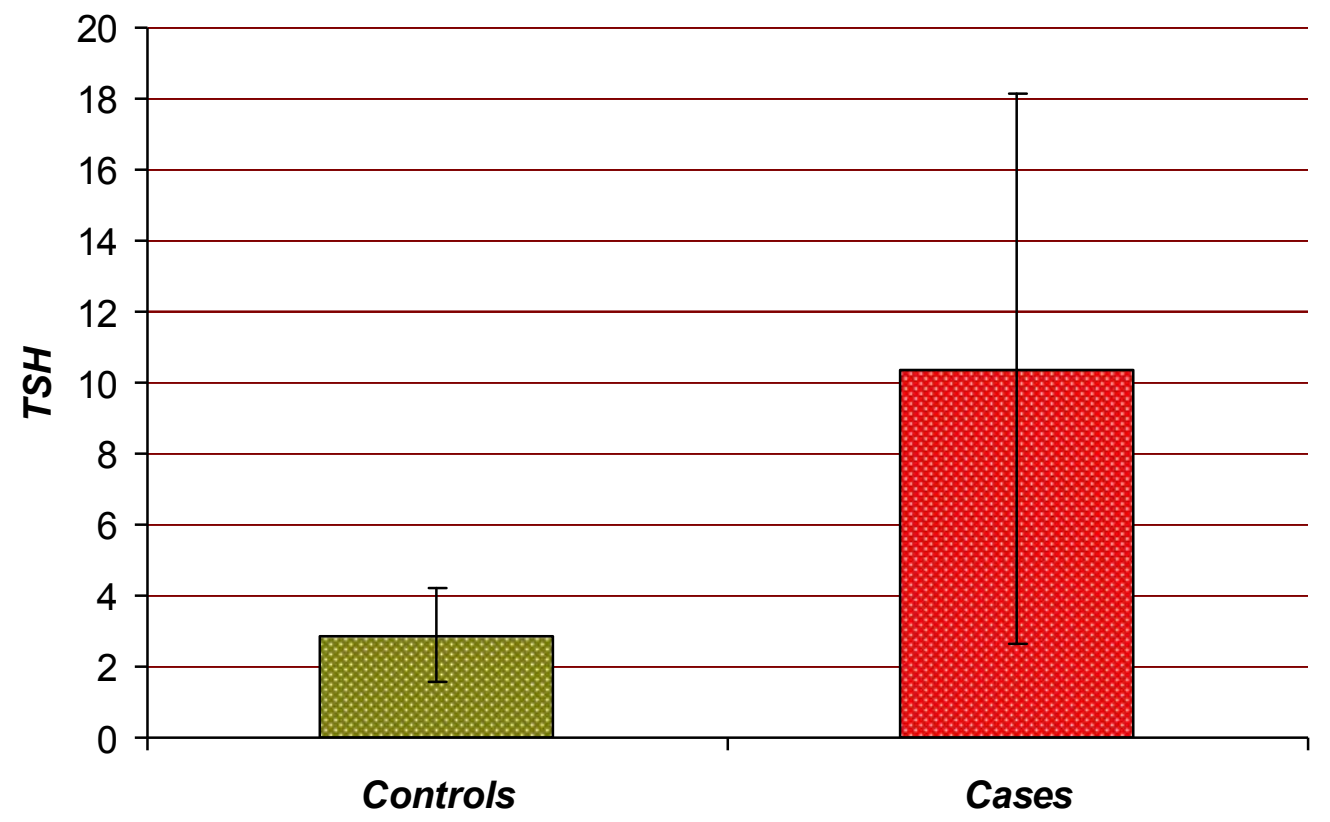

\section{Discussion:}

The thyroid hormones, tri-iodothyronine and tetraiodothynine are insulin antagonists that also potentiate the action of insulin indirectly TRH synthesis decreases in diabetes mellitus. These facts could be responsible for the occurrences of low thyroid hormone levels in some diabetics. The level of TSH in our study was clinically significant in diabetics than in non-diabetics .Result obtained from present study has shown that in type-2 diabetes mellitus, hypothyroidism, which is a better index to monitor type-2 diabetes mellitus, is frequently observed. ${ }^{7}$

In the present study the distribution of age and sex is matching in control and case group. The fasting blood sugar level were elevated in the case group compare to control group which correlates with the results reported earlier ${ }^{8,9.10 \text {, }}$
Failure to recognize the presence of these abnormal thyroid hormone levels in diabetics may be a primary cause of poor management often encountered in some treated diabetics. There is therefore need for routine assay of thyroid hormone on diabetics, particularly those whose conditions are difficult to manage.

\section{References:}

1. WHO 1985 Diabetes mellitus Tecfh.Rep.Ser.No.646.

2. Process S, Delrange E, Vander Borgh T.V., Jamart T, Donckiet JE. Minor alteration in thyroid function test associated with Diabetes mellitus and obesity in outpatient without know thyroid illness". Acta Clin.Blg,. 2001 March April.

3. WHO. 1985. Diabetes Mellitus. Tech.Rep.ser.No. 727.

4. Rajeev Gupta and Anoop Mishra, Author information, "Type-2 diabetes in Indian; 
Regional Disparities"; Dr. J Diabetes Vasc Dis.2007;7(1):12-16.

5. Barzel, US: thyroid decrease after the sixth decade. Curr Ther Endocrinol metab 1997;6:134137.

6. EL Nobre, $\mathbf{Z}$ jorge, $\mathbf{S}$ prates, $\mathbf{C}$ silva and $\mathbf{J J}$ Castro. Profile of thyroid function in a population with type-2 diabetes mellitus"; British endocrine societies, Abstract; 3:P 298

7. David, M. and Ath, MD. Initial management of glycemia in type-2 diabetes mellitus" NEJM, 202; 347(17):1342-1349
8. Bhalla. K, Shukla. R, Gupta. V.P, Pugazhenthi. S. Prabhu. K.M. Glycated protein and serum lipid profile in complicated and uncomplicated NIDDM patient" Ind. J.Clin Biochem.1995, 10:57-6

9. Yegin. A. and Ozben. T. Serum glucated lipoproteins in type-2 diabetes patient with and without complication" Ann. Clin Biochem." 199, 32:459-463

10. Panteghini. M, Cimino, A, Pagani. F, Girelli. A. Nonenzymic glycation of apolipoprotein B in patient with insulin and non-insulin dependent diabetes mellitus" Clin Biochem 1995, 28:587592

Adress of Correspondance: Shekhar Chandra Yadav, Lecturer, Department of Biochemistry, Nobel Medical College, Biratnagar.E-mail:luv4sunrise@yahoo.com 